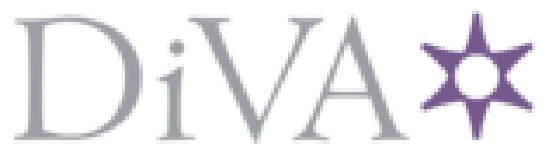

http://www.diva-portal.org

This is the published version of a paper published in .

Citation for the original published paper (version of record):

Engberg, G., Yvell, K., Grehk, M. (2018)

Analysis of the plastic deformation behavior for two austenitic NiCr-steels with different stacking fault energies

International Journal of Computational Physics Series I, : 137-141

https://doi.org/10.29167/A1I1P137-141

Access to the published version may require subscription.

N.B. When citing this work, cite the original published paper.

Permanent link to this version:

http://urn.kb.se/resolve?urn=urn:nbn:se:du-30084 


\title{
Analysis of the plastic deformation behavior for two austenitic NiCr-steels with different stacking fault energies
}

\author{
Göran Engberg ${ }^{1}$, Mikael Grehk ${ }^{2}$ and Karin Yvell ${ }^{1}$ \\ ${ }^{1}$ Dalarna University, Sweden \\ ${ }^{2}$ AB Sandvik Materials Technology, Sweden
}

Email: gen@du.se

\begin{abstract}
Two austenitic stainless steels, with low and medium stacking fault energies (SFE), 20 $\mathrm{mJ} / \mathrm{m}^{2}$ and $30 \mathrm{~mJ} / \mathrm{m}^{2}$ respectively, have been studied by conventional tensile tests and in situ tensile tests in a FEG-SEM equipped for EBSD. High angle boundaries (HAB) and low angle boundaries (LAB) with misorientations $>=10^{\circ}$ and $>=2^{\circ}$ respectively have been determined, and size distributions for the LABs have been derived by linear intercepts. It was found that the size distributions could be described by bimodal lognormal functions. For the steel with highest SFE plastic deformation took place by dislocation slip only while the steel with low SFE deformed by slip and twinning. Using a model for slip based on the evolution of the dislocation density with the generation of dislocations inversely proportional to the mean free distance of slip and recovery of dislocations proportional to the dislocation density the stress strain-curves were analyzed and the results compared with the measured quantities. The mean free distance of slip as evaluated from the stress-strain curve for the steel with the highest SFE correlates very well with the mean size of the LABs intercept. The rate of recovery also gave an expected stress dependence. The stress needed to start deformation twinning was based on the assumption that Shockley partials become completely separated in the slip plane. The thus calculated values for the twinning stress showed an excellent agreement with the observed start of twinning as given by EBSD evaluation of twin boundaries (TB). For the alloy with low SFE both surface grains (in situ test) and bulk grains (from interrupted conventional tests) were studied. The stress needed for slip and twinning of surface grains was, as expected, in the order of 0.5-0.6 times the applied stress.
\end{abstract}

Keywords: Austenitic stainless steels, Electron backscatter diffraction (EBSD), In situ tension test, Grain boundaries, Flow stress model.

\section{Introduction}

Models for the dependence of the flow stress on the dislocation density [1] coupled with models for the evolution of the dislocation density with plastic strain [2] are used to analyse the stressstrain curves of two Cr-Ni alloys with different stacking fault energies. Cross-slip of dislocations has been treated as a thermally activated deformation process, and the model proposed by Friedel [3] and later elaborated by Escaig [4] is used. The onset of deformation twinning is taken from a model proposed by Han et al. [5]. In situ tensile tests in a FEG-SEM equipped with EBSD as well as interrupted conventional tensile tests gave experimental information of the microstructure development which then was correlated to the analysis of the stress-strain curves. The in-situ tests and the conventional tests made it possible to compare the behaviour of surface and bulk grains.

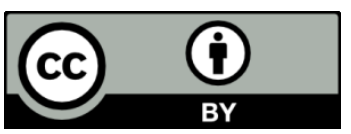

CC-BY Content from this work may be used under the terms of the Creative Commons Attribution 3.0 license. Any further distribution of this work must maintain attribution to the author(s) and the title of the work, journal citation and DOI.

Published under license in Int. J. of Computational Physics Series by NSSEL Publishing. 


\section{Experimental}

Two austenitic stainless Fe-Cr-Ni alloys designed to have stacking fault energies of $20 \mathrm{~mJ} / \mathrm{m}^{2}$ and $30 \mathrm{~mJ} / \mathrm{m}^{2}[7,8]$ have been studied, named alloy S20 and S30 respectively. The composition of the alloys is given in table 1 .

Table 1. Chemical composition (weight-\%).

\begin{tabular}{llllllllllll}
\hline Steel & $\mathrm{Fe}$ & $\mathrm{Ni}$ & $\mathrm{Cr}$ & $\mathrm{Mn}$ & $\mathrm{Si}$ & $\mathrm{Al}$ & $\mathrm{C}$ & $\mathrm{N}$ & $\mathrm{S}$ & $\mathrm{P}$ & $\begin{array}{c}\text { Trace } \\
\text { elements }\end{array}$ \\
\hline $\mathrm{S} 30$ & 49.8 & 31.41 & 18.57 & 0.03 & 0.02 & 0.013 & 0.006 & 0.006 & 0.005 & 0.003 & 0.137 \\
\hline $\mathrm{S} 20$ & 69.4 & 11.7 & 18.75 & 0.04 & 0.02 & 0.004 & 0.006 & 0.007 & 0.005 & 0.003 & 0.065 \\
\hline
\end{tabular}

S30 and S20 have both been studied by in situ tensile testing in a FEG-SEM equipped for EBSD and conventional tensile testing has also been performed. For S20 tensile test samples interrupted at different strains have been studied using EBSD enabling a comparison of deformation structure development in surface versus bulk grains.

The EBSD measurements, performed with a step length of $2 \mu \mathrm{m}$, were used to find high and low angle grain boundaries, HABs and LABs respectively with HABs having misorientation angles $>=10$ degrees and LABs with angles in the range $2-10$ degrees. Twin boundaries, TBs, were also identified. Linear intercepts were used to find the distances between boundaries with misorientation angles $>=2$ degrees. The 2 -degree limit was chosen as it reflected the substructure development as observed on band contrast images well. The distributions of intercept lengths were found to be well described by bimodal log-normal distribution functions, as earlier found for 316L [9]. For S30 the functions were fitted using experimental values $>=10 \mu \mathrm{m}$ and for S20 $>=5 \mu \mathrm{m}$ and in all cases, the maximum cumulative value was fixed to the observed value.

\section{Results and discussion}

\subsection{EBSD measurements}

In alloy S20 deformation twinning was observed to start at a plastic strain of around 0.05 and 0.15 for surface and bulk grains respectively. No twinning was observed in alloy S30 up to a plastic strain of $28 \%$. Some twinning was however observed in a fractured specimen, plastic strain $\sim 34 \%$.

The arithmetic mean sizes, $\mathrm{d}_{\text {small }}$, obtained for the distribution functions of small intercepts were then evaluated. According to Guttieres-Urrutia and Raabe [10] substructure sizes, $d$, have been found to comply with the relation

$\frac{1}{d}=K \frac{\tau}{G b}$

where $\mathrm{K}$ is a constant, $\mathrm{G}$ the shear modulus $(81260 \mathrm{MPa}), \mathrm{b}$ burgers vector $(0.25 \mathrm{~nm})$ and $\tau$ the applied stress. This relation holds well for alloy S30. For S20, however, the sizes obtained at large strains reach the resolution limit of our data (which is somewhere between 3 and $4 \mu \mathrm{m}$ ). For this alloy, we used equation 1 to extrapolate the sizes to higher stresses.

\subsection{Analysis of stress-strain curves}

The dependence of the flow stress on the dislocation density is given by [1]

$\sigma=\sigma_{0}+m \alpha G b \sqrt{\rho}$

where $\sigma_{0}$ are all hardening contributions except deformation hardening, $\mathrm{m}$ the Taylor factor, $\alpha \mathrm{a}$ constant $\sim 0.2$ for fcc and $\rho$ the dislocation density. The evolution of the dislocation density with strain is given by [2] 
$\frac{d \rho}{d \varepsilon_{p l}}=\frac{m}{b} \cdot \frac{1}{L}-\Omega \rho$

where $\varepsilon_{\mathrm{pl}}$ is the plastic strain, $\mathrm{L}$ the mean free distance of dislocation slip and $\Omega$ a remobilization/annihilation parameter. Using equations 2 and 3 we have evaluated $\sqrt{ } \rho$ and $d \rho / d \varepsilon_{\mathrm{pl}}$ by fitting $\sigma_{0}$ and assuming an initial dislocation density of $10^{11} \mathrm{~m}^{-2}$. The mean free distance of slip depends on the grain size, $\mathrm{d}_{\mathrm{HAB}}$, and the dislocation density as

$\frac{1}{L}=\frac{1}{d_{H A B}}+\frac{\sqrt{\rho}}{c_{L}}$

where $c_{L}$ is a constant $>=1$. As $L$ is inversely proportional to $\sqrt{ }_{\rho}, c_{L}$, can be derived from the linear relation between $\mathrm{d} \rho / \mathrm{d} \varepsilon$ and $\sqrt{ } \rho$ at low strains where the influence of recovery $(\Omega \rho)$ is negligible.

In deriving the values for the surface, we have assumed that the flow stress is 0.6 times the values obtained in the conventional tensile tests According to Nabarro et al. [6], the flow stress for surface grains is half that of the flow stress of bulk grains or slightly more depending on the surface properties.

The values obtained for $c_{L}$ were 81.8 and 49.4 for S30, surface and bulk respectively. The corresponding values for S20 are 37.6 and 60.0. The mean free distance of slip, L, can now be calculated. In figure 1 , calculated values of $1 / \mathrm{L}$ are compared to the values of $d_{\text {small }}$ obtained by EBSD. As can be seen, a very good agreement is obtained.

Using the information on $\mathrm{c}_{\mathrm{L}}$ we can now calculate $\Omega$. For $\mathrm{S} 30$ we find that $\Omega$ depends exponentially on stress. This is expected if we assume that cross slip of dislocations is responsible for recovery. By putting

$\Omega=\Omega_{0} \cdot \exp \left(k_{\Omega} \sigma\right)$

we obtain $\Omega_{0}=0.310$ and $\mathrm{k}_{\Omega}=7.17 * 10^{-3}$ for the surface and $\Omega_{0}=0.0783$ and $\mathrm{k}_{\Omega}=6.58^{*} 10^{-3}$ for the bulk.

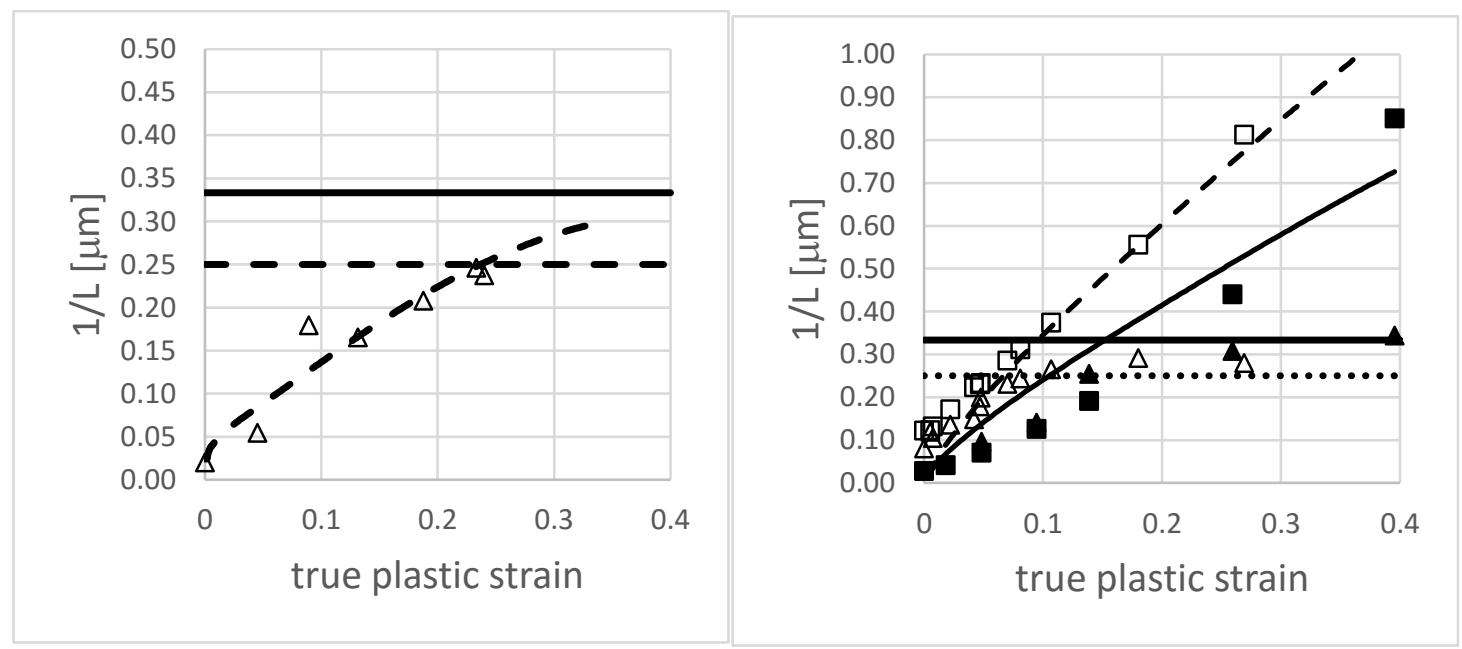

Figure 1. Mean free distance of slip for alloy S30 (left) and S20 (right). The horizontal lines show $\mathrm{L}$ equal to $3 \mu \mathrm{m}$ and $4 \mu \mathrm{m}$. Triangles, $\mathrm{d}_{\text {small. }}$. Squares, regression according to equation 1 .

Surface dashed lines and open markers. Bulk solid line and filled markers.

Alloy S20 behaves quite differently from alloy S30. Here we observe abundant deformation twinning. The stress needed to obtain deformation twinning, $\sigma_{\text {twin, }}$, is given by [5] 


$$
\sigma_{t w i n}=\frac{\mathrm{m}}{m t}\left(\frac{G \cdot b_{p}}{d}+\frac{\gamma}{b_{p}}\right)
$$

where $b_{p}$ is Burgers vector of a Shockley partial, $d$ the dislocation source size, $\gamma$ the stacking fault energy and $\mathrm{mt}$ an orientation factor relating the slip and twinning systems. The onset of twinning is marked with vertical lines in figure 2 . Using equation 6 with $\mathrm{mt}=1$ (the most favored orientation for twinning), we have calculated the twinning stress with the dislocation source size, $d$, equal to $\mathrm{d}_{\text {small. }}$ The influence of the dislocation source size is small, and the stress is dominated by the stacking fault energy. For the bulk, we have used a Taylor factor, $\mathrm{m}=3.1$, and for the surface, where the constraints are much smaller, we have put $\mathrm{m}=2$. The twinning stress is also reduced by a factor of 0.6 as compared to the bulk. In figure 2 the flow stress and twinning stress for both alloys are displayed for both bulk and surface.

Deformation twinning was not observed in the in situ tensile test of alloy S30. In the fractured specimen from conventional tensile test, however, some deformation twins were observed which is in good agreement with the predicted twinning stress.

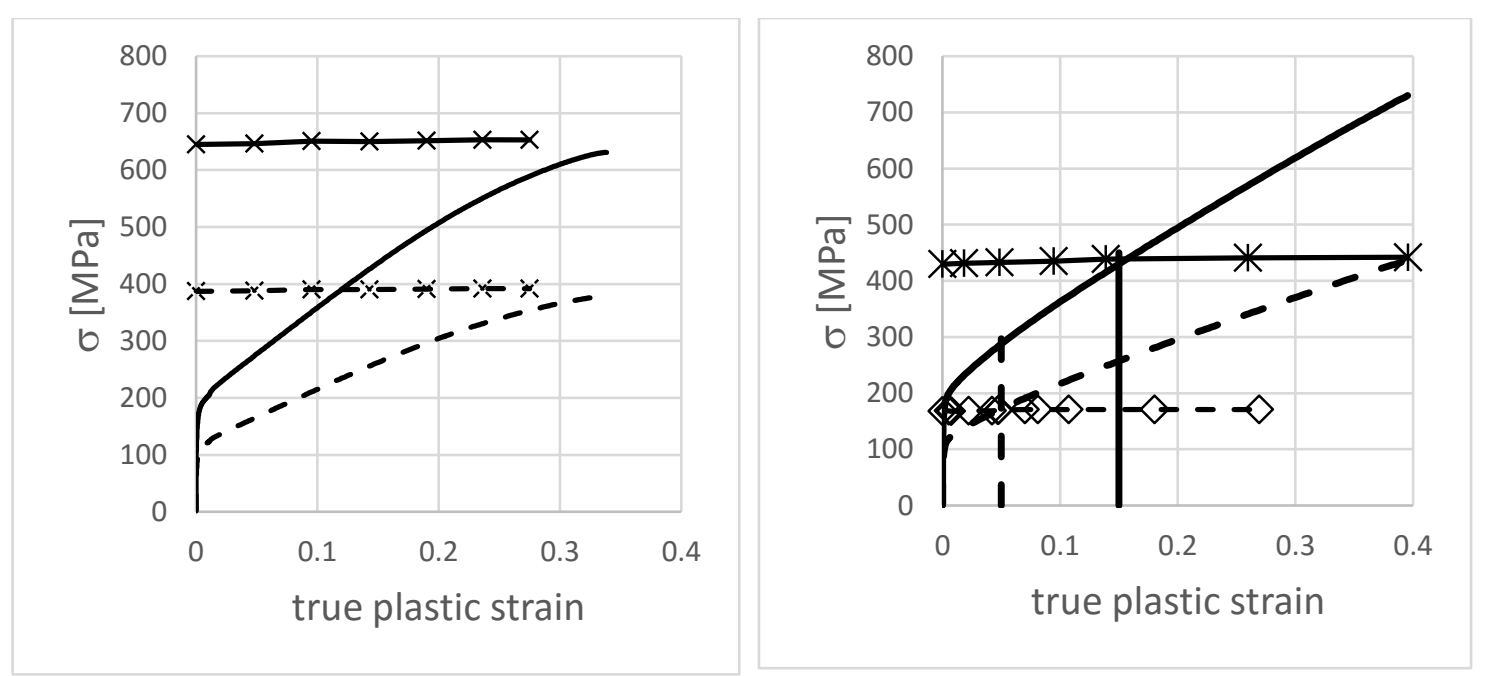

Figure 2. Flow stress and twinning stress for bulk (solid lines) and surface (dashed lines) for alloy S30 (left) and S20 (right). Horizontal lines are twinning stresses, and vertical lines show the onset of TB formation.

As is seen the predicted onset of deformation twinning matches the observations perfectly.

We cannot evaluate $\Omega$ in the same way for alloy S20 as for S30 as the plastic strain is given by both dislocation slip and deformation twinning. Escaig [4] gave an approximate solution of the activation energy for cross-slip at low stresses and if we assume that the resolved shear stress on the cross slip plane is proportional to that on the primary slip plane, $\sigma_{\mathrm{psp}}$, the probability for crossslip can be written as,

$p=p_{0} \exp \left(-\frac{W_{0}}{k T}\right) \cdot \exp \left(\frac{W_{0}}{k T} c_{s} \frac{b \sigma_{p s p}}{\gamma}\right)$

where $\mathrm{k}$ Boltzmann's constant, $\mathrm{T}$ the temperature in Kelvin and $\mathrm{c}_{\mathrm{s}}$ and $\mathrm{p}_{0}$ constants. $\mathrm{W}_{0}$ is a function of the equilibrium distance between two Shockley partials at zero applied stress. If we assume that all parameters in equation 7 except $\mathrm{W}_{0}$ and $\gamma$ are equal for both alloys we can evaluate $\Omega_{0}$ and $\mathrm{k}_{\Omega}$ for alloy S20 from the corresponding values for S30. We then obtain $\Omega_{0}=5.52 * 10^{-58}$ and $\mathrm{k} \Omega_{\Omega}=1.56^{*} 10^{-2}$ for the bulk and $\Omega_{0}=2.18^{*} 10^{-57}$ and $\mathrm{k}_{\Omega}=1.70^{*} 10^{-2}$ for the surface. With the applied stresses for S20 this gives very small values of $\Omega$ (of the order $10^{-50}-10^{-60}$ ), and we thus expect recovery by cross-slip to be negligible. The dislocation evolution with combined slip and twinning is given by 


$$
\frac{d \rho}{d \varepsilon_{p l}}=\left[\frac{m}{b}\left(\frac{1}{d_{H A B}}+\frac{\sqrt{\rho}}{c_{L}}\right)-\Omega \rho\right] \cdot\left(1-\frac{d \varepsilon_{t w i n}}{d \varepsilon_{\text {slip }}}\right)
$$

where $\varepsilon_{\text {twin }}$ is plastic strain caused by twinning and $\varepsilon_{\text {slip }}$ by dislocations. The fraction twinned material, $f_{\text {twin }}$, with the twin shear $1 / \sqrt{2}$, is given by

$f_{\text {twin }}=\varepsilon_{\text {twin }} \cdot m \sqrt{2}$

If recovery is negligible equation 8 can be integrated directly and using equation 9 we can now calculate the twinned volume fraction. We have also attempted to measure the fractions using 158 points in a square grid and counting the number of points that fall within a deformation twin. The values, $4.4 \%$ at $18 \%$ plastic strain for the surface and $4.4 \%$ at $25.5 \%$ for the bulk, are quite close to the calculated fractions, $3.3 \%$ and $3.9 \%$ respectively, but it should be pointed out that the measurements are quite difficult due to the large step size used for the images.

\section{Conclusions}

The size distributions of intercept lengths, evaluated using EBSD identification of boundaries with a misorientation $>=2$ degrees, are well described using bimodal log-normal distribution functions. The arithmetic mean of the distribution for small sizes is in good agreement with the mean free distance of slip as evaluated from the stress strain-curves.

In the alloy with a stacking fault energy of $30 \mathrm{~mJ} / \mathrm{m}^{2}$ annihilation/remobilization of dislocations is found to depend exponentially on stress, as expected if recovery of dislocations is assumed to be governed by cross slip.

In the alloy with a stacking fault energy of $20 \mathrm{~mJ} / \mathrm{m}^{2}$ deformation twinning was found to start at stress levels predicted by the model proposed by Han et al. [5]. The model for cross-slip as proposed by Escaig [4] showed that recovery of dislocations by this mechanism was not possible in this alloy. This enabled the evaluation of the evolution of the volume fraction of deformation twins with strain, in good agreement with the microstructure observations.

The flow stress for surface grains was found to be a factor of 0.6 times that of bulk material. Using a Taylor factor of 2 for the surface and 3.1 for the bulk gave an excellent prediction of the twinning stress and the incidence of twinning as observed in the in the situ tensile tests and conventional tensile tests respectively.

\section{Acknowledgements}

AB Sandvik Materials Technology, Dalarna University, Regional Development Council of Dalarna, Regional Development Council of Gävleborg, County Administrative Board of Gävleborg, Swedish Steel Producers' Association and Sandviken City are thanked for financial support.

\section{References}

[1] Taylor G J 1934 Proc Royal Soc of London 145362

[2] Bergström Y 1983 Rev Powder Metall Phys Ceram 2 79-265

[3] Friedel J 1964 Dislocations (Pergamon)

[4] Escaig B 1968 J Phys 29225

[5] Han W Z, Zhang Z F, Wu S D and Li X 2008 Phil Mag 88:24 3011-3029

[6] Nabarro F R N, Basinski Z S and Holt D B 1964 Adv in Phys 13 193-323

[7] Lu S, Hu Q-M, Johansson B and Vitos L. 2011 Acta Mater 59 5728-5734

[8] Lu,J, Hultman L, Holmström E, Antonsson K H, Grehk M, Li W, Vitos L and Golpayegani A 2016 Acta Mater $11139-46$

[9] Yvell K, Grehk T M and Engberg G 2016 Materials Characterization 122 14-21

[10] Guttieres-Urrutia I and Raabe D 2012 Acta Mater 60 5791-802 